\title{
Accurate Non-Intrusive Residual Bandwidth Estimation in WMNs
}

\author{
Ismail Cem Atalay, Yunus Sarikaya, Ozgur Gurbuz, Ozgur Ercetin \\ Faculty of Engineering and Natural Sciences, Sabanci University, Istanbul, Turkey \\ Email: \{ismailcem, sarikaya, ogurbuz, oercetin\}@ sabanciuniv.edu
}

\begin{abstract}
The multi-access scheme of 802.11 wireless networks imposes difficulties in achieving predictable service quality in multi-hop networks. In such networks, the residual capacity of wireless links should be estimated for resource allocation services such as flow admission control. In this paper, we propose an accurate and non-intrusive method to estimate the residual bandwidth of an 802.11 link. Inputs from neighboring network activity measurements and from a basic collision detection mechanism are fed to the analytical model so that the proposed algorithm calculates the maximum allowable traffic level for this link. We evaluate the efficiency of the method via OPNET simulations, and show that the percent estimation error is significantly lower than two other prominent estimation methods, bounded only between 2.5-7.5\%. We also demonstrate that flow admission control is successfully achieved in a realistic WMN scenario. Flow control through our proposed algorithm keeps the unsatisfied traffic demand bounded and at a negligibly low level, which is less than an order of magnitude of the other two methods.
\end{abstract}

\section{INTRODUCTION}

With the proliferation of 802.11 based wireless networks, people begin to expect predictable and better service quality from wireless networks. A key step in the provision of better service quality is to estimate traffic handling capacities of the wireless network links/paths. The difference between the network link/path's capacity and its current throughput identifies the additional user demand that can still be met under current conditions. This difference is known as residual bandwidth and was previously discussed in the literature [1], [2], [3] within the framework of ad hoc wireless networks.

The problem of accurate estimation of residual bandwidth without causing overhead in 802.11 based wireless multi-hop networks is still an open problem involving wireless medium characteristics dynamically changing according to the user traffic patterns and the channel conditions. In order to obtain a good estimate of residual bandwidth, the transmission activity in the channel should be identified accurately without causing disruption to the network operation.

In this paper, we provide a generalized analysis of the wireless link capacity under various network conditions considering the effects of different link rates, packet sizes, channel impairments and hidden nodes. This approach differs from those available in the literature, since it combines real measurements with analytical calculations. In fact, this is why our residual bandwidth estimation method is so powerful; i.e., percent estimation error remains between 2.5-7.5\%. Since measurements are made by overhearing DATA-ACK messages and by inserting very small time-stamps on retransmitted

This work was supported in part by European Commission Netadded STREP (SST5-CT-2006-030960). packets, the algorithm is non-intrusive for network operations. The computational complexity is low, and thus, the algorithm can be implemented easily on each wireless node. We also demonstrate how flow admission control is carried out by estimating the path residual bandwidth through the proposed method. The rest of the paper is organized as follows: Section II provides a brief summary of the earlier studies on residual bandwidth estimation. Section III presents an overview of proposed algorithm with a step-by-step description of the estimation process and introduces the network monitoring scheme providing inputs to the algorithm. Section IV describes interrelated time-sharing and contention models for saturated link. Section V explains the traffic and collision models for unsaturated competing links. Section VI provides performance analysis for the proposed algorithm. Section VII concludes the paper by providing a summary of our contribution.

\section{BACKGROUND}

Primary access method of IEEE 802.11 is by means of CSMA/CA based Distributed Coordination Function (DCF). A summary of 802.11 DCF operation can be found in [4].

There are two residual bandwidth estimation approaches in 802.11 based networks: 1) active or intrusive, 2) passive or non-intrusive methods. In active methods, probe packets are used either to saturate wireless links or to explore link characteristics. In the former case, the residual bandwidth is estimated by detecting the delay variation at saturation [1] [2]. In the latter case, "hello" packets are inserted to neighboring traffic [3] to circulate local available bandwidth information so that contention levels are deduced and then used in residual bandwidth estimation. The drawback of active methods is their significant overhead due to extra probe packets.

Passive approaches to residual bandwidth estimation are non-intrusive, as no additional packets are inserted into the system. One popular method that we call as "passive listen" [5], [6] is based on listening channel activity to deduce channel idleness ratio. This ratio is adjusted via a smoothing constant [5] or a weight factor [6] against the backoff and collision related overestimation and then multiplied with data rate to estimate the residual bandwidth. However, due to 802.11 network characteristics, an empirical weight factor causes significant inaccuracies in estimation process. Another passive technique, called "time measurement" method, [7] is based on measuring the time spent for successful DATA packet delivery. This measured delay is then normalized according to packet size and its reciprocal value is taken as total bandwidth. The residual bandwidth of the wireless link is obtained by subtracting current load from this total bandwidth value. Both 
of these techniques do not model changing system dynamics as saturation is approached. Therefore, they are unable to take into account capacity reducing effects of inter-link and inter-flow competition. Two other approaches by Gao et al [8] and Garetto et al [9] concentrate on estimating available throughput by non-intrusive analytical methods that do not rely on network measurements. However the former one [8] has some drawbacks such as limiting collision events to hidden node interference and ignoring channel errors. The latter study [9] follows an indirect approach by analytically producing network metrics that, when appropriately used, decrease unfairness and starvation for competing flows in a wireless multihop network. It also assumes lossless channel condition and presents a reactive method that regulates dominant flows rather than a proactive one that estimates true residual capacity.

\section{Algorithm Overview}

Our algorithm is designed to run in the transmitter node of a directed link (named as the primary link from now on) for which we aim to calculate the residual bandwidth. This primary link belongs to a contention domain that is defined as the set of all directed 802.11 links in the network such that only one link from this set can be active at a given time [10]. All members of this contention domain excluding the primary link itself are called as competing links. The main inputs of our algorithm are the number of competing links, the number of successful packet deliveries per unit time on competing links and the primary link packet failure rate. The number of competing links and their level of traffic are obtained by monitoring overheard DATA-ACK messages on the channel (refer to section III-B). Meanwhile, the packet failure rate is deduced from a basic collision detection scheme already existing in the literature [11] (refer to section III-C). Our

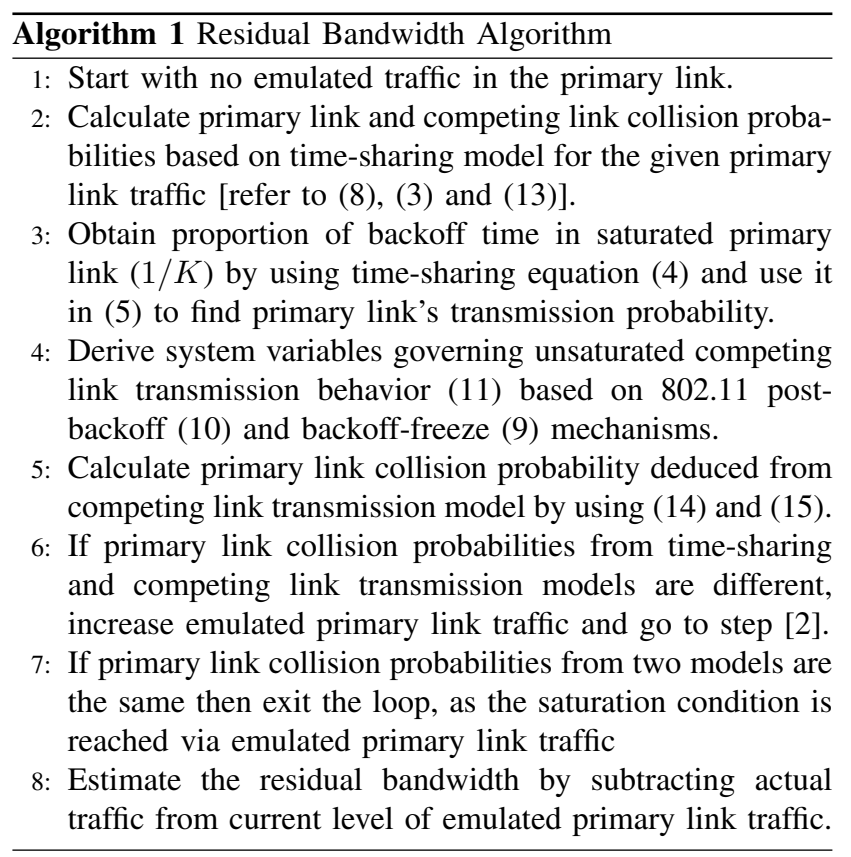

proposed algorithm's operation is summarized in Algorithm 1. In essence, our algorithm mimics the active residual bandwidth estimation methods (saturating the wireless link via probe packets), and estimates the residual bandwidth without actually transmitting packets.

\section{A. Network Model}

In this paper, we focus on Wireless Mesh Networks operating on a single frequency channel where there are multiple contention domains. Let $N_{p \text { saturation }}$ be the number of successful packet transmissions per unit-time over the primary link under saturation, and let $f_{p}$ be the current throughput on this link in bits/sec and $S$ be the average payload size in bits. The residual bandwidth of primary link, $R B_{p}$, is defined as

$$
R B_{p}=S \times N_{p, \text { saturation }}-f_{p},
$$

The assumptions used in our analysis are given as follows :

- Primary link packet failure rate $\left(p_{p}^{f}\right)$ is fixed for all traffic levels.

- Packet failure rate for the competing links is the same as primary link packet failure rate.

- There is no maximum retransmission limit.

- The probability of having an empty MAC buffer after the post-backoff stage is equal to the probability of having an empty MAC buffer.

\section{B. Wireless Network Activity Monitoring Scheme}

Let $N_{l, i}$ be the total number of successful packet deliveries per unit-time in the $i$ 'th competing link, and $L-1$ be the actual number of competing links where $L$ is the total number of wireless links in the contention domain. $N_{l, i}$ and $L-1$ can be obtained by counting the number of overheard DATA-ACK messages per unit time, and by classifying the DATA-ACK messages according to their source and destination addresses. Since our proposed network monitoring scheme only deals with the overheard DATA-ACK packets, it requires a small computational and memory resource.

\section{Packet Failure Rate Estimation}

In order to accurately estimate the residual capacity of the primary link, packet delivery errors caused by collisions and packet failures should be distinguished. Therefore, we define $p_{p}^{f}$ and $p_{p}^{c, \text { measured }}$ as the packet failure probability and measured collision probability for the primary link respectively. The collision related packet delivery errors are strongly correlated to the primary link traffic level. However, packet failures caused by wireless channel impairments and by interference from hidden nodes are mostly independent from the primary link traffic load. If we can measure $p_{p}^{f}$ for current status of network traffic activity, this value might be directly used in equations governing primary link's saturation.

In order to obtain packet failure probability $p_{p}^{f}$, we use the collision detection method proposed in [11]. This algorithm utilizes a non-intrusive scheme based on transmission history of the nodes operating in the same contention domain and is embedded in our proposed estimation algorithm as an accurate 
way of measuring $p_{p}^{c, \text { measured }}$. Since primary link packet error probability $p_{p}^{e, \text { measured }}$ (that is directly measurable in all 802.11 transmitter nodes) is a combination of the collision and packet failure probabilities, packet failure probability $p_{p}^{f}$ can easily be derived from two measured quantities as follows,

$$
p_{p}^{f}=\frac{p_{p}^{e, \text { measured }}-p_{p}^{c, \text { measured }}}{1-p_{p}^{c, \text { measured }} .}
$$

\section{PRimary Link System Analysis}

In order to determine the utilization of channel as a shared resource among primary and competing links, we first model the channel access in a unit time duration under saturation condition. According to 802.11 DCF operation, unit time duration in a saturated link is split between transmission, collision and backoff periods. Let $N_{p}$ be the number of successful packet transmissions per unit-time over the primary link. We first determine the proportion of time spent by transmission attempts based on $N_{p}$ and $N_{l, i}$ values. The average number of transmission attempts over the primary link in unit-time is $\frac{N_{p}}{1-p_{p}^{e}}$ and the average number of transmission attempts over any competing link in unit time is $\frac{N_{l, i}}{\left(1-p_{l, i}^{e}\right)}$, where $p_{p}^{e}$ and $p_{l, i}^{e}$ are defined as the probability of packet delivery error on the saturated primary link and the i'th competing link respectively. Let us also assume that primary link is a member of an endto-end path $P$ over which we would like to send traffic.

We assume that packet failure rate $p_{p}^{f}$ is obtained through the procedure explained in section III-C. $p_{p}^{e}$ and $p_{l, i}^{e}$ that are the primary and $i$ 'th competing link packet error probabilities at saturated primary link condition are calculated as

$$
\begin{aligned}
p_{p}^{e} & =p_{p}^{f}+p_{p}^{c}-p_{p}^{f} p_{p}^{c}, \\
p_{l, i}^{e} & \approx p_{p}^{f}+p_{l, i}^{c}-p_{p}^{f} p_{l, i}^{c},
\end{aligned}
$$

where $p_{p}^{c}$ and $p_{l, i}^{c}$ are collision probabilities on the saturated primary link and the $i$ 'th competing link, respectively. Since we do not make collision detection for the competing links, we use $p_{p}^{f}$ as an approximation for their packet failure rate.

Let $T_{t r}$ be the average time period between a DATA packet's transmission start event and reception of its acknowledgment. $T_{t r}$ for the average payload size $S$ is calculated according to [12] by considering 802.11 MAC overhead and physical rate.

On a saturated link, the idle portion of time is only composed of backoff times. Let $\bar{B}_{p}$ be the mean back-off time per attempt on the primary link. Thus, the idle time under saturation can be expressed as the product of $\bar{B}_{p}$ and the average number of transmission attempts per unit-time $\frac{N_{p}}{\left(1-p_{p}^{e}\right)}$. To account for overlapping transmissions, we only consider one half of the total duration spent for collisions. We also need to consider transmissions occurring in competing links belonging to the traffic path $P$. Thus, one unit time on the primary link under saturation is shared according to the following equation:

$$
\begin{aligned}
& \frac{N_{p}}{1-p_{p}^{e}}\left(1-\frac{p_{p}^{c}}{2}\right)\left(T_{t r}+D\right)+\sum_{i=1}^{L-1}\left[\frac{N_{l, i}}{1-p_{l, i}^{e}}\left(1-\frac{p_{l, i}^{c}}{2}\right)\left(T_{t r}+D\right)\right]+ \\
& \sum_{i \in P}\left[\frac{N_{p}}{1-p_{l, i}^{e}}\left(1-\frac{p_{l, i}^{c}}{2}\right)\left(T_{t r}+D\right)\right]+\frac{N_{p}}{1-p_{p}^{e}} \bar{B}_{p}=1,
\end{aligned}
$$

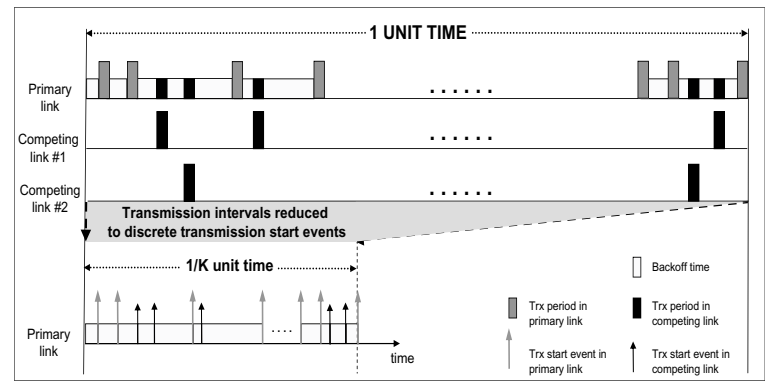

Fig. 1. 1/K units of idle backoff time in one unit time of network events.

where $D$ is DIFS period. Let $p_{p}^{t}$ and $p_{l, i}^{t}$ be the transmission probabilities for primary and the $i$ 'th competing link respectively when the primary link is in saturation condition. We express $p_{p}^{t}$ in terms of average number of transmission attempts per unit time on the primary link. As demonstrated in Figure 1, we equivalently consider only the idle backoff periods on the primary link and approximate the transmissions as instantaneous events. Also assume that time is slotted and the slot length is $\mu$. This approach is similar to the one used in the seminal work by Bianchi [4], since the contention and collision behavior of $802.11 \mathrm{DCF}$ can be modeled as a discrete-time random process. Let the proportion of idle time spent in a unit time be $1 / K$. Figure 1 illustrates this setup by providing a typical snapshot of 802.11 network events. Also notice that there can be a new transmission attempt only when the channel is idle. Therefore, the transmission probability for the saturated 802.11 link is defined as the ratio of the number of transmission start events per unit time to the number of time-slots in $1 / K$ time units where all transmission start events are supposed to occur. Thus, $p_{p}^{t}$ is

$$
p_{p}^{t}=\frac{N_{p}}{\left(1-p_{p}^{e}\right) \frac{1 / K}{\mu}}=\frac{\mu \times K \times N_{p}}{1-p_{p}^{e}},
$$

Accordingly, $1 / K$ units of idle backoff time is equal to the product of mean backoff time per attempt and the number of transmission attempts in unit time as follows,

$$
\frac{1}{K}=\frac{N_{p}}{1-p_{p}^{e}} \bar{B}_{p}
$$

The mean back-off time per attempt $\left(\bar{B}_{p}\right)$ can be derived in terms of $p_{p}^{e}$ and the minimum contention window size $W$, by observing the binary exponential backoff behavior. According to 802.11 MAC, the mean backoff time increases exponentially at each re-transmission, e.g., at $k$ th re-transmission the mean backoff time is $\frac{\mu\left(2^{k} W-1\right)}{2}$. Then, the mean backoff time per packet, $\bar{B}_{\text {total }}$ is,

$$
\begin{aligned}
\bar{B}_{\text {total }} & =\sum_{i=0}^{\infty}\left(1-p_{p}^{e}\right)\left(p_{p}^{e}\right)^{i}\left(\frac{1}{2} \sum_{k=0}^{k=i}\left(2^{k} W-1\right) \mu\right) \\
& =\frac{\mu W}{2\left(1-2 p_{p}^{e}\right)}-\frac{\mu}{2\left(1-p_{p}^{e}\right)}
\end{aligned}
$$


In the primary link, the average number of attempts per single successful packet delivery is $1 /\left(1-p_{p}^{e}\right)$. By using this and (7), we have

$$
\bar{B}_{p}=\bar{B}_{\text {total }}\left(1-p_{p}^{e}\right)=\frac{\mu W\left(1-p_{p}^{e}\right)}{2\left(1-2 p_{p}^{e}\right)}-\frac{\mu}{2} \approx \frac{\mu W\left(1-p_{p}^{e}\right)}{2\left(1-2 p_{p}^{e}\right)}
$$

where the last equality follows from $W>>1$ and $\frac{1-p_{p}^{e}}{1-2 p_{p}^{e}}>1$.

If we insert $\bar{B}_{p}$ into (6) we obtain,

$$
p_{p}^{e}=\frac{1}{2}-\frac{\mu \times W \times K \times N_{p}}{4}
$$

Therefore, (8) gives us a basic relationship between the packet error probability of the primary link $p_{p}^{e}, K$ and the target variable $N_{p}$ that we would like to determine eventually.

\section{Competing Link System Analysis}

In this section, we modify our analysis to take into account the unsaturated link behavior of the competing links. There has been recent interest in understanding the behavior of unsaturated 802.11 links, and [13] provides an analysis using a state-transition scheme based on finite load source model. Based on this approach, we let $q$ be the probability of having an empty MAC buffer after the last packet transmission ends. According to 802.11 DCF standard, if the MAC buffer of a node is empty, the transmitter enters the post-backoff stage, where the system waits for a backoff time randomly chosen between $[0, W-1]$ slots. After this post-backoff stage, MAC buffer is checked, and if there has been any packet arrival, next packet is directly transmitted. Let $q^{\prime}$ be the probability of having an empty MAC buffer after the post-backoff stage. The transmitting node enters a waiting stage with probability $q^{\prime}$ until a new arrival. Upon an arrival, transmitting node senses the medium. If the medium is idle (with probability $p_{\text {idle }}$ ), then packet is immediately transmitted. However, if the medium is sensed busy (with probability $1-p_{i d l e}$ ), then the system proceeds with standard backoff. In order to have a model that does not rely on a specific packet arrival pattern, we assume that $q^{\prime} \simeq q$, since the mean post-backoff time is significantly smaller than the mean packet inter-arrival time for a great majority of possible traffic arrival rates.

The probability of having an empty MAC buffer in the transmitter of $i$ 'th competing link $q_{i}$ can be determined by using Little's theorem as $q_{i}=1-\lambda_{i} E\left[S T_{i}\right]$ where $\lambda_{i}$ is the average packet arrival rate and $E\left[S T_{i}\right]$ is the expected service time on $i$ 'th competing link.

We can identify four different cases under which $E\left[S T_{i}\right]$ needs to be calculated. Let $B_{l, i}, B_{l p b, i}$, and $F_{i}$ be the mean backoff, mean post backoff and mean backoff freeze durations per successful delivery for $i$ 'th competing link, respectively. Also let $\overline{S T}_{k, i}$ and $P(k)_{i}, k=1, \ldots, 4$ be average service time and the probability of occurrence of each of the cases, respectively. Therefore,

- Case 1: Non-empty buffer after transmission; $\overline{S T}_{1, i}=$ $B_{l, i}+F_{i}+T_{t r}, P(1)_{i}=1-q_{i}$.

- Case 2: Non-empty buffer after post-backoff stage; $\overline{S T}_{2, i}=B_{l p b, i}+F_{i}+T_{t r}, P(2)_{i}=q_{i}\left(1-q_{i}\right)$.
- Case 3: Empty buffer after post-backoff, channel busy; $\overline{S T}_{3, i}=\frac{1}{\lambda_{i}}+B_{l, i}+F_{i}+T_{t r}, P(3)_{i}=q_{i}^{2}\left(1-p_{i d l e}\right)$.

- Case 4: Empty buffer after post-backoff, channel idle, transmit directly; $\overline{S T}_{4, i}=\frac{1}{\lambda_{i}}+F_{i}+T_{t r}, P(4)_{i}=q_{i}^{2} p_{i d l e}$. Note that the proportion of time the channel is idle per unit time interval is given as $1 / K$, so $p_{\text {idle }}=1 / K$. Backoff freeze occur at competing links when there is another ongoing transmission on the channel. The total proportion of time where backoff freeze occur in $i$ 'th competing link, $B_{i}^{f r}$ is thus defined as,

$$
\begin{aligned}
& B_{i}^{f r}=\left(1-q_{i}\right)\left[\frac{N_{p}}{1-p_{p}^{e}}\left(1-\frac{p_{p}^{c}}{2}\right) T_{t r}+\sum_{j=1, j \neq i}^{L-1}\left(\frac{N_{l, j}}{1-p_{l, j}^{e}}\left(1-\frac{p_{l, j}^{c}}{2}\right) T_{t r}\right)\right] \\
& +\left(1-q_{i}\right)\left[\sum_{j \in \text { path }, j \neq i}\left(\frac{N_{p}}{1-p_{l, j}^{e}}\left(1-\frac{p_{l, j}^{c}}{2}\right) T_{t r}\right)\right]
\end{aligned}
$$

Then $F_{i}$ is the ratio of $B_{i}^{f r}$ to the number of successful packet deliveries in unit time, i.e., $\frac{B_{i}^{f r}}{N_{l, i}}$.

Meanwhile, the mean backoff and mean post-backoff times for competing links can be determined in a way similar to the derivation of mean backoff time per attempt $\overline{B_{p}}$.

$$
\begin{aligned}
\bar{B}_{l, i} & =\frac{\mu W\left(1-p_{l, i}^{e}\right)}{2\left(1-2 p_{l, i}^{e}\right)} \\
\bar{B}_{l p b, i} & =\frac{\mu(W-1)}{2}\left(1-p_{l, i}^{e}\right)+p_{l, i}^{e} \bar{B}_{l, i}
\end{aligned}
$$

Thus, the average service time, $E\left[S T_{i}\right]$ is calculated as,

$E\left[S T_{i}\right]=\left(1-q_{i}\right) \overline{S T}_{1, i}+q_{i}\left(1-q_{i}\right) \overline{S T}_{2, i}+q_{i}^{2}\left(1-p_{i d l e}\right) \overline{S T}_{3, i}+q_{i}^{2} p_{\text {idle }} \overline{S T}_{4, i}$

If we make appropriate simplifications and insert (10) into $q_{i}=1-\lambda_{i} E\left[S T_{i}\right]$ equation, we obtain the following quadratic equation for $q_{i}$ in terms of $\lambda_{i}, F_{i}, p_{i d l e}, \bar{B}_{l, i}, \bar{B}_{l p b, i}$ and $T_{t r}$.

$\left[\lambda_{i} \bar{B}_{l, i}\left(1-\frac{1}{K}\right)+1-\lambda_{i} \bar{B}_{l p b, i}\right] q_{i}^{2}+\left[\lambda_{i}\left(\bar{B}_{l p b, i}-\bar{B}_{l, i}\right)+1\right] q_{i}+$

$\lambda_{i}\left(\bar{B}_{l, i}+F_{i}+T_{t r}\right)-1=0$

In (11), it is possible to mathematically prove that for the quadratic equation in the form $a q_{i}^{2}+b q_{i}+c=0, a$ is always positive for all possible values of $\mathrm{K}$ between 1 and $+\infty$ and $c$ is always negative. Therefore discriminant $\Delta=b^{2}-4 a c$ is always positive giving two distinct real roots for $q_{i}$. Since $\lambda_{i}$ is the average packet arrival rate for any of the competing links, we simply have $\lambda_{i}=N_{l, i}$. Therefore, all the coefficients in (11) can be written in terms of $K, N_{p}, p_{l, i}^{e}, p_{p}^{e}, N_{l, i}$ and $q_{i}$ is solvable in terms of these variables.

Next, we determine the probability of collision on the unsaturated competing links. Similar to what we did in equation (5), transmission probability for $i$ 'th competing link is

$$
p_{l, i}^{t}=\frac{\mu \times K \times N_{l, i}}{1-p_{l, i}^{e}},
$$

Therefore, the collision probability of the $i$ 'th link is,

$$
p_{l, i}^{c}=1-\left[\prod_{j=1, j \neq i}^{L-1}\left(1-p_{l, j}^{t}\right)\right]\left(1-p_{p}^{t}\right)
$$


By using (3), $p_{l, i}^{e}$ term in (12) can be expressed in terms of $p_{l, i}^{c}$ in equation (13). By recursively solving (13) and (12), we obtain $p_{l, i}^{c}$ for every link in terms of $p_{p}^{t}, K$ and $N_{l, i}$. Since $p_{p}^{t}$ is related to $p_{p}^{c}$ by (5) and (3), we have $p_{l, i}^{c}$ as a function of $p_{p}^{c}, K$ and $N_{l, i}$ as desired.

We also need to calculate collision probability of the primary link, $p_{p}^{c}$ by taking into account competing links transmission behavior. $p_{p}^{c}$ can be written as the probability of observing a transmission in at least one of the competing links given that a transmission already occurred on the primary link. Due to special transmission behavior of unsaturated links (transmit directly after sensing idle vs. transmit after backoff), for every $i$ 'th competing link unit backoff time is divided into two disjoint regions corresponding to different pair-wise collision behavior between primary and $i$ 'th competing link. By using the definition of transmission probability in (5), the probability that the $i$ 'th competing link transmits after backoff/post-backoff is

$$
p_{\text {backoff }, i}^{t}=\left(1-P(4)_{i}\right) \frac{\mu \times K \times N_{l, i}}{\left(1-p_{l, i}^{e}\right)},
$$

and the probability that the $i$ th competing link transmits directly is

$$
p_{\text {direct }, i}^{t}=P(4)_{i} \frac{\mu \times K \times N_{l, i}}{\left(1-p_{l, i}^{e}\right)} .
$$

where $P(4)_{i}=\frac{q_{i}^{2}}{K}$. Thus, the pair-wise probability of collision between the primary link and the $i$ 'th competing link is obtained as follows,

$$
\begin{aligned}
& p_{p, i}^{c}=p_{\text {backoff }, i}^{t}\left(1-P(4)_{i}\right)+p_{\text {direct }, i}^{t} P(4)_{i} \\
& =\left[\left(\frac{q_{i}^{2}}{K}\right)^{2}+\left(1-\frac{q_{i}^{2}}{K}\right)^{2}\right] \frac{\mu \times K \times N_{l, i}}{1-p_{l, i}^{e}}
\end{aligned}
$$

Finally, $p_{p}^{c}$ is derived from pair-wise collision probabilities as

$$
p_{p}^{c}=1-\prod_{i=1}^{L-1}\left(1-p_{p, i}^{c}\right) .
$$

Since $q_{i}$ 's in (11) are already derived in terms of $K, N_{p}$, $p_{l, i}^{c}$ and $p_{p}^{c}$, it is possible to recursively solve for $p_{p}^{c}$ from (15) (for a given $K$ and $N_{p}$ ) by using $p_{l, i}^{c}$ from (13).

\section{Performance Analysis}

The performance of the proposed residual bandwidth estimation algorithm is evaluated in comparison to passive listen [6] and time measurement [7] methods via simulations. Simulations are performed in the OPNET environment, where the nodes constitute a wireless mesh network with IEEE $802.11 \mathrm{~g}$ based air interface. The RTS/CTS feature of 802.11 is disabled and the packet arrivals in the source nodes follow Poisson distribution. Poisson packet arrival assumption was previously used in some of the prominent approaches [4] [9] [13] that directly contributed to the idea development of our algorithm. Simulations have been run for 60 seconds, when maximum percent deviation from steady-state residual bandwidth estimate has fallen below $1 \%$. The weight factor

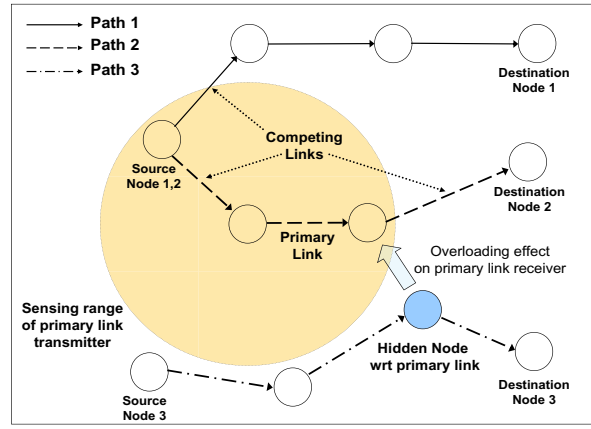

Fig. 2. Simple Wireless Mesh Network

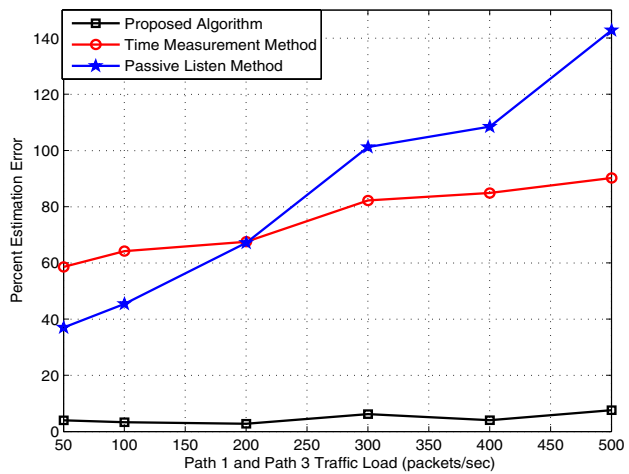

Fig. 3. Percent estimation error for simple WMN

for the passive listen method has been set to its optimal value for the corresponding primary link data rate.

First, we consider a simple scenario depicted in Figure 2 where we have three network paths and link data rates are randomly selected from $802.11 \mathrm{~g}$ rates such as $6,24,36$, and $54 \mathrm{Mbps}$. Data payload sizes for the flows using these paths are chosen arbitrarily as one of these three values (2048, 4196 or 8192 bits). Note that we have a hidden node that interferes with primary link's receiver but gets undetected by the transmitter. Throughout the simulation, primary link residual bandwidth estimates obtained by the algorithms are recorded while the traffic on path 1 and path 3 is increased from 0 up to the level when the primary link reaches saturation. Traffic load on path 2 (that the primary link is a member of) remains constant. Figure 3 shows the percent estimation error which is the absolute difference between the actual and estimated residual bandwidth divided by the actual bandwidth value. As seen from this figure, our proposed algorithm significantly outperforms the other methods with percentage error of only 2.5-7.5\%.

Secondly, we demonstrate the application of our residual bandwidth estimation method for flow admission control in a realistic WMN scenario. This time, we consider a large network where 50 nodes are randomly placed in a $1000 \mathrm{~m}$ 


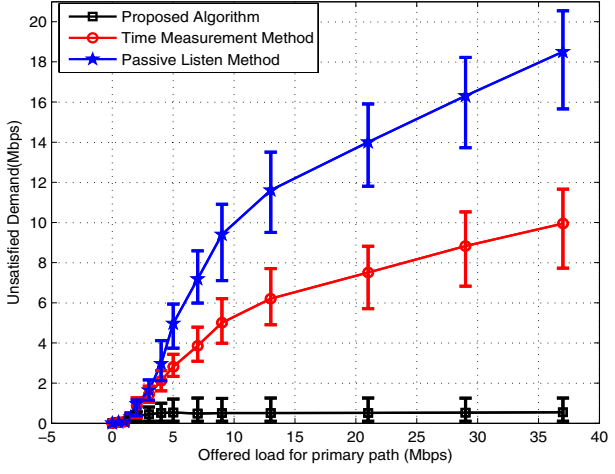

Fig. 4. Unsatisfied demand due to overflows for primary path

$\mathrm{x} 1000 \mathrm{~m}$ area. Both transmission and sensing ranges are set equal to $200 \mathrm{~m}$. Channel impairments are modeled according to free-space path loss and flat Rayleigh fading assumptions. The received power through the flat Rayleigh fading channel is determined according to exponential distribution whose mean value is the received power after the path loss effect.

In this setting, 10 source/destination node pairs are randomly selected out of 50 nodes constituting 10 different endto-end paths. One of these paths has been randomly chosen as our primary path on which we will apply flow control by using residual bandwidth estimation algorithms. The source nodes in the remaining 9 paths establish competing flows by sending a predetermined level of traffic to their destination nodes. For flow control purposes, standard AODV protocol is modified, so the residual bandwidth of the path can be computed from the estimates of the residual bandwidth of the links along the primary path. Residual bandwidth values estimated in the transmitter node of each link on the primary path are embedded into the AODV route reply messages and relayed back to the source node. The source node calculates the endto-end path residual bandwidth by picking up the minimum of received link residual bandwidth values. Flow admission control is then carried out according to the residual capacity, i.e. when the estimated path residual bandwidth reaches zero, source node of the primary path ceases to admit new flows. Figure 4 shows the results of the flow control for three residual bandwidth estimation methods obtained from ten different runs of above scenario.

The curves for unsatisfied traffic demand denote the average rate of lost traffic (in Mbps) due to buffer overflows on primary path, and the upper and lower bounds of error bars indicate maximum and minimum levels observed for each method. With our proposed estimation algorithm, the unsatisfied demand is kept as bounded and negligibly low as the source node does not admit new flows to the system when end-toend path saturation is reached. The passive listen and time measurement algorithms overestimate the residual bandwidth, and thus, the flow control based on the estimations of these algorithms admits more traffic than the network can handle. Consequently, at least one order of magnitude of more traffic remains unsatisfied with these methods.

\section{CONCLUSION}

In this paper, we have presented a novel non-intrusive method to estimate residual bandwidth of 802.11 wireless networks under a generic WMN scenario considering hidden nodes and wireless channel impairments. The method makes use of the measurements on link activity for building analytical models of collision and traffic behavior. It is shown by simulation experiments that the proposed algorithm provides accurate estimates, with an error margin around 2.5-7.5\%. When residual bandwidth estimates are utilized in flow admission control for WMNs, the proposed method outperforms two prominent non-intrusive methods via more accurate knowledge of available network capacity. To the best of our knowledge, the proposed algorithm is the first residual capacity estimation method for WMNs that can simultaneously handle channel impairments, collisions and interference effects. It is practically implementable in all types of 802.11 based nodes and would be applicable to a variety of network configurations, under different traffic loads and characteristics.

\section{REFERENCES}

[1] M. N. Nielsen, K. Ovsthus, and L. Landmark, "Field trials of two 802.11 residual bandwidth estimation methods," MASS 2006, pp. 702-708.

[2] C. Sarr, C. Chaudet, G. Chelius, and I. G. Lassous, "Improving accuracy in available bandwidth estimation for ieee 802.11-based ad hoc networks," MASS 2006, pp. 517-520.

[3] Y. Yang and R. Kravets, "Contention-aware admission control for ad hoc networks," IEEE Transactions on Mobile Computing, vol. 04, no. 4, pp. 363-377, 2005.

[4] G. Bianchi, "Performance analysis of the ieee 802.11 distributed coordination function," IEEE Journal on Selected Areas in Communications, vol. 18 , no. 3, pp. 535-547, Mar 2000.

[5] K. Xu, K. Tang, M. Rajive Bagrodia; Gerla, and M. Bereschinsky, "Adaptive bandwidth management and qos provisioning in large scale ad hoc networks," MILCOM 2003, vol. 2, pp. 1018-1023.

[6] L. Chen and W. Heinzelman, "Qos-aware routing based on bandwidth estimation for mobile ad hoc networks," IEEE Journal on Selected Areas in Communications, vol. 23, no. 3, pp. 561-572, March 2005.

[7] S. Shah, K. Chen, and K. Nahrstedt, "Dynamic bandwidth management for single-hop ad hoc wireless networks," PerCom 2003, pp. 195-203.

[8] Y. Gao, D.-M. Chiu, and J. C. Lui, "Determining the end-to-end throughput capacity in multi-hop networks: Methodology and applications," SIGMetrics/Performance06.

[9] M. Garetto, T. Salonidis, and E. W. Knightly, "Modeling per-flow throughput and capturing starvation in csma multi-hop wireless networks," INFOCOM 2006.

[10] V. P. Mhatre, H. Lundgren, F. Baccelli, and C. Diot, "Joint mac-aware routing and load balancing in mesh networks," 2007 ACM CoNEXT, no. 19.

[11] J.-H. Yun and S.-W. Seo, "Novel collision detection scheme and its applications for ieee 802.11 wireless lans," Elsevier Computer Communications, pp. 1350-1366, 2007.

[12] D. Kliazovich and F. Granelli, "Cross-layer congestion control in ad hoc wireless networks," Ad Hoc Networks, vol. 4, pp. 687-708, Nov. 2006.

[13] G. R. Cantieni, Q. Ni, C. Barakat, and T. Turletti, "Performance analysis under finite load and improvements for multirate 802.11," Computer Communications, vol. 28, pp. 1095-1109, Jun. 2005. 